\title{
Mobile Sensor Node Deployment Strategy by using Graph Structure based on Estimation of Communication Connectivity and Movement Path
}

\author{
Koji Kawabata ${ }^{1}$, Tsuyoshi Suzuki ${ }^{2}$ \\ Department of Information and Communication \\ Engineering, Tokyo Denki University \\ Tokyo, Japan
}

\begin{abstract}
We propose a multiple-mobile sensor node (MSN) deployment strategy that considers wireless communication quality and operation time of underground wireless sensor networks. After an underground disaster, it is difficult to perform a rescue operation because the internal situation cannot be confirmed. Hence, gathering information using a teleoperated robot has been widely discussed. However, wireless communication is unstable and the corresponding wireless infrastructure to operate the teleoperated robot is unavailable underground. Therefore, we studied the disaster informationgathering support system using wireless sensor networks and a rescue robot. In this study, the movement path information of the teleoperated robot is fed to MSNs in a graph structure. MSNs are deployed in the underground environment by adding an evaluation of communication quality and operation status to a given graph structure. The simulation was evaluated in an assumed underground environment. The results confirmed that the wireless communication quality between each MSN was maintained and energy consumption was balanced during the deployment.
\end{abstract}

Keywords-Wireless sensor networks; deployment strategy; communication connectivity

\section{INTRODUCTION}

Gathering information in disaster areas is very important for assessing the situation, avoiding secondary disasters, and reducing disasters [1-5]. In general, bird's-eye image information gathered by unmanned air vehicles (UAVs) and artificial satellites is useful for understanding post-disaster situations. However, in an underground space in the city where such UAVs cannot gather information, it is difficult to ascertain the extent of the damage, which is important for avoiding secondary disasters. In addition, rescue teams cannot organize a suitable rescue plan for underground spaces owing to the lack of sufficient information. In these situations, the rescue team go underground to gather disaster information. The information is then shared within the on ground and underground teams for efficient and cooperative rescue work. However, when the communication infrastructure is broken due to damage, rescue teams cannot cooperate closely because of the disconnection. Therefore, the rescue team must work underground without the complete knowledge of the situation and face the added risk of secondary disasters.
From studies based on past accident analysis, researchers have recently focused on a disaster information-gathering method using a wireless sensor network (WSN) and a rescue robot in closed areas. The WSN consists of spatially distributed sensor nodes (SNs) to cooperatively monitor environmental conditions such as temperature, sound, vibration, pressure, motion, etc. The WSN is then enabled to provide wireless communication without the existing infrastructure. In a closed area, it is constructed using a rescue robot. Therefore, an information-gathering method by constructing the communication infrastructure in a disaster area using a WSN has been discussed [6,7]. However, the scope of application is limited to outside the disaster area, and studies assuming a closed space have not been reported.

Information gathering by rescue robots and disaster rescue support systems is effective. The use of robot technology can reduce the activity burden on rescue workers. Rescue robots are often remotely controlled by considering the impact on the disaster area and work safety. When the robot is operated remotely, it is possible to support rescue operations in spaces where people cannot easily enter, such as closed spaces, narrow spaces, and underwater. The connectivity and stability of communication are very important during remotely controlled operations. Owing to the closed environment, wireless communication is often unstable underground, as compared to the outdoors. Hence, degradation of wireless communication due to disturbances such as fading and shadowing is more likely to occur underground than outdoors.

Therefore, we studied the information-gathering system using teleoperated robots and WSNs, as observed in Fig. 1 [810]. In this system, a WSN is constructed using a mobile sensor node (MSN). This system responds quickly to network disconnection. This system reports that the end-to-end throughput was maintained and an effective WSN was constructed. However, the autonomous deployment of each MSN is unavailable in this system. In environments where wireless communication is unstable, such as the underground, the MSN must be placed in a position that provides a stable wireless relay to maintain the communication connection for the teleoperated robot. In addition, the MSN has limited energy because the power supply is a battery. Hence, it is important for the WSN that there is no network disconnection when the MSN stops operating. 


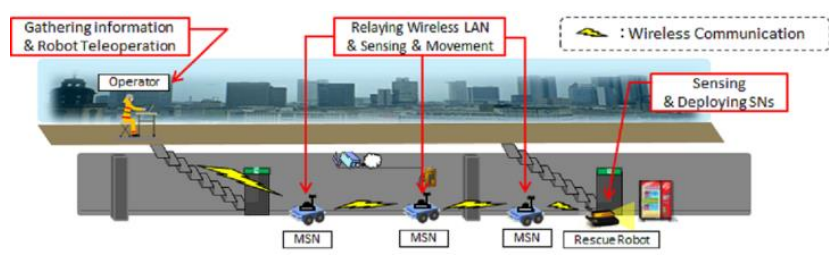

Fig. 1. Disaster Information-Collecting Support System using a RemoteControl Robot and WSNs.

Certain studies reported on relocating the MSN to be positioned where communication quality was evaluated in the field of the WSN [11,12]. Furthermore, in the field of multiagent systems, cooperative control of multiple robots based on communication quality has been reported [13,14]. These studies utilized the received signal strength indicator (RSSI) for communication quality. Saitou [13] was reported that the deployment control which made always uniform RSSI between each robot was performed. Zhong [14] was presented that the distributed control which establish and maintain RSSI between the robot was performed. By evaluating RSSI, it is demonstrated that the MSN can be autonomously deployed at the position where wireless communication between MSNs is stabilized in WSNs.

In general, WSNs are required to operate for a long time and hence, energy efficient relocation methods have been reported $[15,16]$. The relocation of the SNs at the appropriate movement distances reduced the network energy consumption and maximized the coverage level. Conversely, studies have proposed that each MSN is placed at the position where the energy consumption between each MSN is balanced [17,18]. By applying energy costs, such as energy consumption and the standard deviation of energy consumption between MSN to coverage to MSN deployment model, the overall energy of WSNs is balanced. These studies conclude that long-term operation is possible by reducing and balancing the energy consumption of each SN.

Therefore, the proposed MSN deployment strategy considers an underground space, which is positioned where the communication quality between MSNs and the operating status of the MSN are evaluated. We then evaluated the communication quality between MSNs and energy balance of each MSN using a simulation.

\section{DEPLOYMENT STRATEGY OF MSN}

Our proposed deployment strategy requires a graph structure construction and autonomous operation of multiple MSNs. In a disaster environment where the situation is unknown, it is difficult to remotely control a robot and multiple MSNs simultaneously. Therefore, it is desirable to position MSNs where communication quality in the environment is maintained while moving autonomously. However, a fully autonomous operation of the robot may malfunction in the disaster-area environment.

Hence, the robot is operated remotely such that when a movement target is given by the operator, the movement path information is shared by the WSN. The MSN is autonomously operated by following the robot based on its movement path information. In addition, each MSN evaluates communication quality and its own operation status while moving on the path and is placed on the path based on the evaluation.

\section{A. Precondition}

In the information-gathering system, the network is constructed in the order of the operator, MSNs, and robot in a straight line. Furthermore, a change in the network topology during the construction of the WSN may disconnect the network when the communication path is changed. Particularly, wireless communication in an underground space is unstable, and it is likely for the network to disconnect. Therefore, the network topology of this system does not change.

Information sharing with neighboring terminals is necessary for autonomous operation of MSNs. The information shared with the neighboring terminal is (1) the threedimensional absolute coordinate information, (2) a remaining energy, and (3) path information of robot movement. The MSN is operated to follow the robot's path. Each MSN acquires the coordinate information of the neighboring terminals to accurately follow the robot. Further, the remaining energy is used to evaluate the operating status. Because the WSN is constructed in a straight line, each MSN shares information with two terminals. Furthermore, the information shared is regularly updated. The update frequency of these information differs. The information of (1) and (2) is regularly updated 1 seconds because MSN follow the robot's path accurately. The information of (3) is regularly updated 10 seconds because the different received information is assumed.

\section{B. Construction Guidelines for a Graph Structure}

The walls and ceilings are less likely to collapse during a disaster because the underground space has a strong structure and an excellent seismic resistance [19]. Therefore, it is unlikely that the environmental structure will change significantly. However, it is difficult to use the map when obstacles occur due to the disaster situation. To place multiple MSNs depending on the environmental situation, it is necessary to analyze the environment. Because SLAM (Simultaneous Localization and Mapping) is often used to analyze the environment by a mobile robot, the teleoperated robot also uses SLAM to collect information while analyzing the environment, which builds the detailed environment with a metric map. In contrast, when MSN requires the robot to follow its path, it is easier to control the robot by specifying a characteristic position on the movement path. An example of a characteristic position is the inflection point of a passage.

Therefore, a topological map is used to represent the environmental information given to each MSN. The topological map represents the environment in a graph structure as shown in Fig. 2. A topological map enables adaptive movement control to the environment while saving computational resources of the MSN. The topological map can plan a path by providing coordinate information to a node [20,21]. The nodes are set to a position where the communication quality is likely to fluctuate and the MSN is relatively difficult to move. For example, the node is set to a coordinate that starts and ends at a stair, such as a corner or a crossroad. The edge is set on the direct movement path between the robot and MSN. 


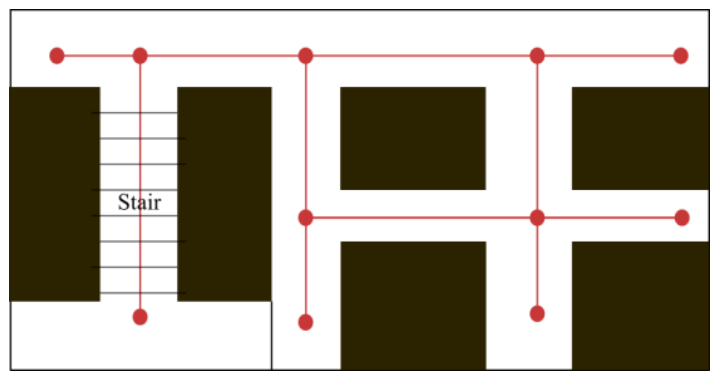

Fig. 2. Processing Flow of the Deployment Algorithm.

The topological map is constructed using sensors (sonar and LRF (Laser Range Finder)) mounted on the teleoperated robot. When a characteristic position is observed by the robot sensors, the node is set to absolute coordinates of the robot at that position. By setting the cost of the edge to the communication quality, the MSN can move along the path where communication is stable. Because each MSN shares the same map, the graph structure is sent to each MSN and the base station.

\section{Deployment Algorithm for MSN}

1) Algorithm overview: Fig. 3 shows the MSN deployment algorithm. Each MSN periodically measures the communication quality between neighboring MSNs to evaluate the communication quality. When detecting a decrease in the quality, the detected MSN evaluates the remaining energy between neighboring MSNs. Based on the evaluation value, the MSN with the large remaining energy, i.e., the MSN with a sufficient operating time moves preferentially. The MSN to be moved calculates the moving direction from the shortest path information and moves. It stops when the communication quality recovers. With this algorithm, each MSN is operated autonomously while maintaining the communication quality between the MSNs and balancing the energy of each MSN.

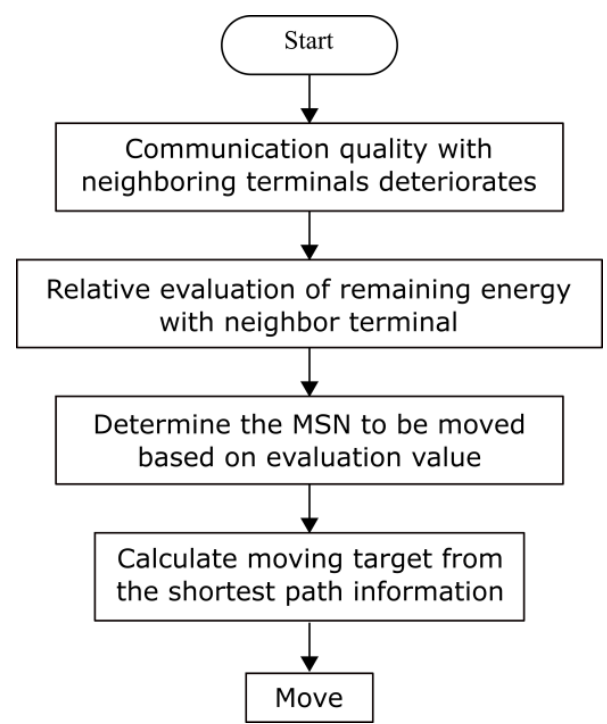

Fig. 3. Topological Map Assuming an underground Space. The Node is Set to the Coordinates at the Start and end of a Stair, such as a Corner or a Crossroad.
2) Path planning: The path planning based on the topological map determines the candidate of the deployment position by calculating the shortest path between each MSN. To perform remote control between the operator and the robot, it is necessary to place each MSN at a position that maintains communication connectivity on the movement path based on the map. Therefore, map-based path planning is necessary. It determines the candidate of the deployment position by calculating the shortest path. The algorithm used in path planning uses Dijkstra's algorithm [22]. Path planning is performed at regular intervals to always obtain the latest movement path information. The update frequency is 10 seconds, which is the same as the interval for acquiring the movement path information from neighboring MSN. Each MSN selects the nodes up to neighboring MSN by path planning and stores the coordinates of the node in the data memory. The MSN moves by referring to the coordinates from its own data memory.

3) Evaluation of communication quality: The communication index uses RSSI. Each MSN sets a threshold to detect a decrease in the RSSI. The MSN determines whether to relocate based on the threshold. When an MSN detects that at least one RSSI has fallen below the threshold, it shifts to the process of evaluating the remaining energy. The MSN evaluates the remaining energy for the neighboring MSN connected to the communication path with the worst RSSI. If the RSSI of the multiple communication paths fall below the threshold, the MSN prioritizes recovering the minimum RSSI. The RSSI is maintained by relocating the MSNs to a position where the RSSI does not always decrease.

4) Evaluation of remaining energy: Each MSN evaluates the remaining energy with the neighboring MSN. The MSN with the higher remaining energy moves preferentially such that the energy consumption of each MSN is uniform. The evaluation of the remaining energy is relative and is performed by the MSN that detects a decrease in RSSI. The relative evaluation of the remaining energy is calculated using

$\varepsilon=\frac{|E(i)-E(j)|}{\max (E(i), E(j))}(0 \leq \varepsilon \leq 1)$

$E(i)$ is the remaining energy of the MSN performing the evaluation process and $E(j)$ is the remaining energy of the neighboring MSN that is to be evaluated. If the value of $\varepsilon$ is closer to 1 , the difference between the remaining energy of $E(i)$ and $E(j)$ is large. Further, both the MSNs that are to be evaluated are determined the moving MSN based on the evaluation value. The MSN is determined according to the following conditions:

- When $\varepsilon \leq \varepsilon_{t h}$, the evaluated MSN moves.

- When $\varepsilon>\varepsilon_{t h}$, the MSN of $\max (E(i), E(j))$ moves. The other MSN does not move.

$\varepsilon_{t h}$ is the threshold of the evaluation value of the remaining energy. After the MSN to be moved is determined by this condition, the MSN starts moving. 
5) Movement control of MSN based on the topological map: The MSN moves based on the coordinates of the nodes obtained in the shortest path to recover the RSSI. Fig. 4 demonstrates the MSN relocation due to the decrease in RSSI. RSSI $_{\text {th }}$ is the RSSI threshold between neighboring terminals. As previously mentioned, when RSSI ${ }_{\text {th }}$ was lower than RSSI, the MSN to move was determined by the relative evaluation of the remaining energy. Furthermore, the moving MSN refers to the coordinates from the data memory used to recover the RSSI. These coordinates are defined as sub-targets. The MSN moves by calculating the moving direction from the relative coordinates with the sub-target. When the MSN reaches the sub-target, the next coordinate is referred to from the data memory and again set as a new sub-target. It then calculates the moving direction and moves. If the RSSI exceeds the threshold before the MSN reaches the sub-target, the MSN stops at that position.

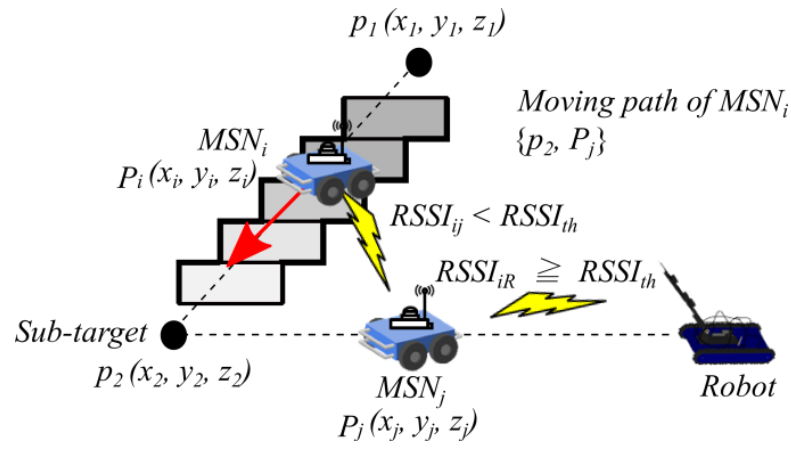

Fig. 4. $M S N_{i}$ Recovers RSSI by Running Down Stairs based on the Shortest Path.

\section{SimULATIONS}

The simulation of the proposed method was verified in a three-dimensional coordinate-system environment without obstacles. The simulator used the open-source network simulator ns-3 [23], which has a model of wireless communication and radio wave propagation of mobile communication bodies. In the simulation, the base station is defined as Sink and the teleoperated robot as Leader.

The simulation conditions are shown in Table I. The network topology is constructed linearly with Sink-MSN1MSN2 - MSN3 - Leader and is not changed during the simulation. Network settings are set according to the wireless communication protocol of the information collection system which is being discussed. Radio wave propagation is based on a noise model. Adding a noise model to the simulation helps in verifying the method in a realistic environment. The method's reliability can also be improved. Fig. 5 depicts the graph structure used in the experiment. The nodes and edges in this experiment were verified using a pre-built graph structure. The nodes are assigned IDs and have coordinates. The cost of an edge is the linear distance between the nodes. The nodes were set at these positions in accordance with the universal underground structure in Japan. The simulation, we demonstrated the effectiveness of the proposed method by performing two verification experiments.
TABLE. I. SimUlation SETting

\begin{tabular}{|c|c|c|}
\hline Parameter & Unit & Value \\
\hline Number of MSN & & 4 \\
\hline $\begin{array}{l}\text { Initial position } \\
\text { (Sink } \\
\text { MSN1, } \\
\text { MSN2, } \\
\text { MSN3, } \\
\text { Leader) }\end{array}$ & (m) & $\begin{array}{l}(-0.8,-0.8,0) \\
(-0.6,-0.6,0) \\
(-0.4,-0.4,0) \\
(-0.2,-0.2,0) \\
(0,0,0)\end{array}$ \\
\hline Link-layer protocol & & IEEE802.11g \\
\hline Network/Transport protocol & & UDP/IP \\
\hline Radio-wave propagation loss model & & Free space \\
\hline Noise model & & Nakagami $m$ distribution \\
\hline Node velocity & $(\mathrm{m} / \mathrm{s})$ & 0.2 \\
\hline Tx power & $(\mathrm{dBm})$ & 16 \\
\hline RSSI threshold & $(\mathrm{dBm})$ & -50 \\
\hline
\end{tabular}

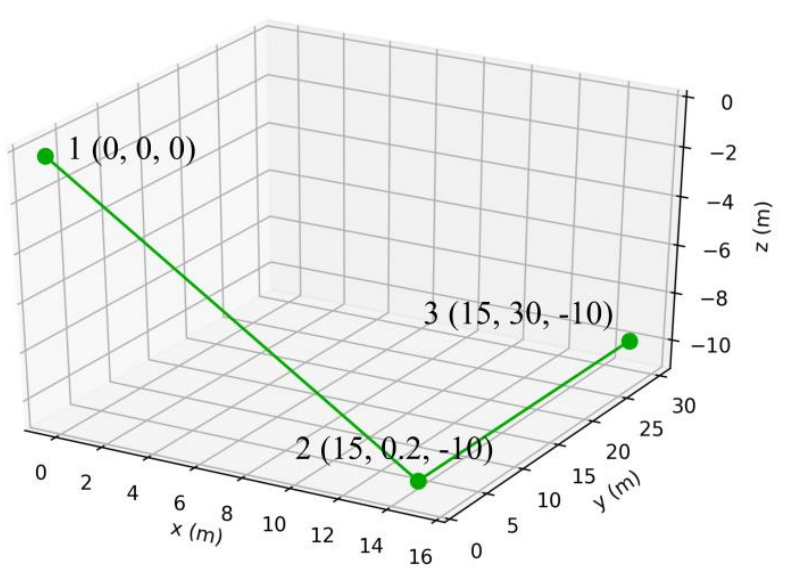

Fig. 5. Graph Structure using Experiment. These Nodes are Assigned IDs and have Coordinates.

\section{A. Evaluation of RSSI by Deployment Algorithm}

This experiment evaluated RSSI between each MSN over time. The Leader moves in the order of nodes with IDs 1, 2, and 3. Each MSN moves through the node of the shortest path calculated by path planning.

The results are shown in Fig. 6 and 7. Fig. 6 represents the RSSI between the deployed MSNs when threshold of the RSSI is $-50 \mathrm{dBm}$, and. Fig. 7 shows the movement trajectory of each MSN. In Fig. 6, Sink-MSN1, MSN1-MSN2, MSN2MSN3, MSN3 - Leader are the observed RSSI between each MSN. Sink-MSN1 (10 s MA), MSN1-MSN2 (10 s MA), MSN2-MSN3 (10 s MA), MSN3-Leader (10 s MA) are the calculated RSSI by moving average every 10 seconds. Each RSSI was maintained at approximately $-35 \mathrm{dBm}$ for SinkMSN1, approximately $-50 \mathrm{dBm}$ for MSN1-MSN2 and MSN2 - MSN3, and approximately $-62 \mathrm{dBm}$ for MSN3 - Leader. Each RSSI was observed to converge in 300 s. In Fig. 7, each MSN was deployed according to the graph structure. Each MSN moved within MSN1 at $(1.165,0.016,-0.777)$, MSN2 at (8.486, 0.113, -5.658), and MSN3 at $(15,1.4,-10)$. 


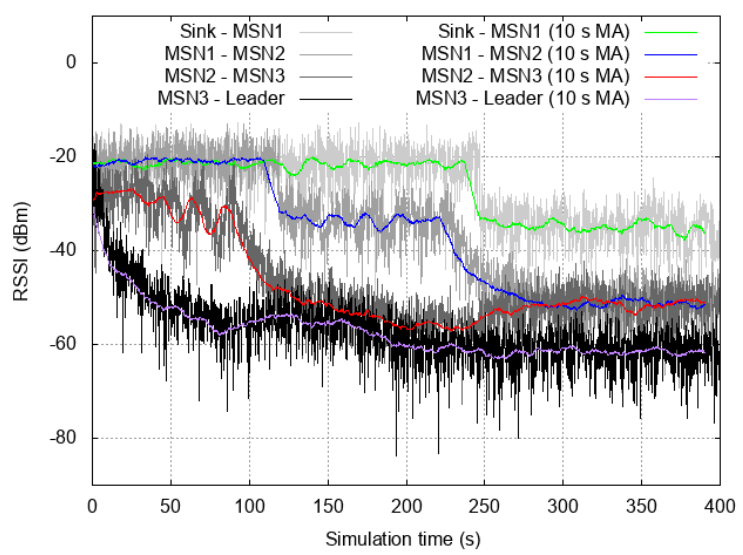

Fig. 6. RSSI During Deployment over Time.

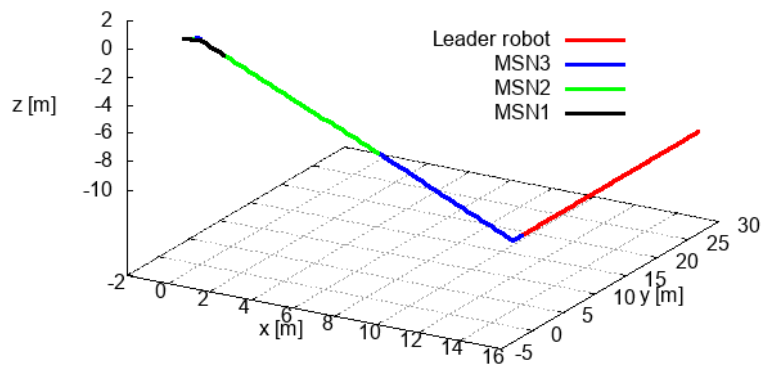

Fig. 7. Movement Trajectory of each MSN.

\section{B. Comparison of Moving Energy Consumption}

This experiment was compared with the deployment algorithm used in this study when the remaining energy was not evaluated. We evaluated the total mobile energy consumption when each MSN was deployed. Based on the results, we performed relative evaluation of the remaining energy by (1) and evaluated the balanced energy between MSN. Table II lists the setting conditions for calculating energy consumption. The total energy consumption of each MSN was calculated by the sum of the energies of wireless communication, movement, and sensor. The movement of the Leader is the same as in the previous experiment.

The results are listed in Tables III and IV. Table III demonstrates the change in each MSN energy consumption when the process of evaluating the remaining energy is applied to the deployment algorithm and when it is not applied. Table IV shows the results of relative evaluation of the remaining energy of each MSN from Table III using (1). In Table III, the difference in energy consumption of each MSN increased by $+1245 \mathrm{~J}$ for MSN1, but decreased by $-1145 \mathrm{~J}$ for MSN2 and -1195.2 J for MSN3. In Table IV, the method that evaluates RSSI and remaining energy has a smaller relative evaluation value. If the relative evaluation value is closer to 0 , the difference between the remaining energy of both MSNs is more balanced.

Here, we examined whether a difference appears in the convergence position by these comparison methods. The examination results are shown in Fig. 8. The red dot represents MSN1 at $(1.165,0.016,-0.777)$, MSN2 at $(7.155,0.095$, 4.770), and MSN3 at (15, 0.6, -10). The blue dot represents
MSN1 at $(1.331,0.017,-0.888)$, MSN2 at $(7.654,0.102$, $5.103)$, and MSN3 at (15, 0.2, -10).

TABLE. II. ENERGY CONSUMPTION SETTING

\begin{tabular}{|l|l|l|}
\hline Parameter & Unit & Value \\
\hline Initial energy & (J) & 100,000 \\
\hline Controller supply voltage & (V) & 5.0 \\
\hline Motor supply voltage & (V) & 15.0 \\
\hline Idle current & (A) & 0.60 \\
\hline Tx current & (A) & 0.85 \\
\hline Rx current & (A) & 0.65 \\
\hline Motor current & (A) & 3.22 \\
\hline Sensor energy consumption & (J) & 2.5 \\
\hline \multicolumn{1}{|c|}{$\varepsilon_{t h}$} & & 0.04 \\
\hline
\end{tabular}

TABLE. III. ENERGY CONSUMPTION OF MSNS

\begin{tabular}{|l|l|l|}
\hline \multirow{2}{*}{$\begin{array}{l}\text { MSN } \\
\text { No. }\end{array}$} & \multicolumn{2}{|l|}{ Moving energy consumption $(\mathbf{J})$} \\
\cline { 2 - 3 } & Proposed method & $\begin{array}{l}\text { Proposed method (the remaining energy is } \\
\text { not evaluated) }\end{array}$ \\
\hline 1 & 5989.58 & 4744.58 \\
\hline 2 & 9702.49 & 10847.89 \\
\hline 3 & 15321.59 & 16516.79 \\
\hline
\end{tabular}

TABLE. IV. ENERGY CONSUMPTION OF MSNS

\begin{tabular}{|l|l|l|}
\hline \multirow{2}{*}{} & \multicolumn{2}{|l|}{ Relative evaluation value of remaining energy } \\
\cline { 2 - 3 } & $\begin{array}{l}\text { Proposed } \\
\text { method }\end{array}$ & $\begin{array}{l}\text { Proposed method } \text { (the remaining energy } \\
\text { is not evaluated) }\end{array}$ \\
\hline MSN1 - MSN2 & 0.040 & 0.065 \\
\hline MSN2 - MSN3 & 0.063 & 0.064 \\
\hline
\end{tabular}

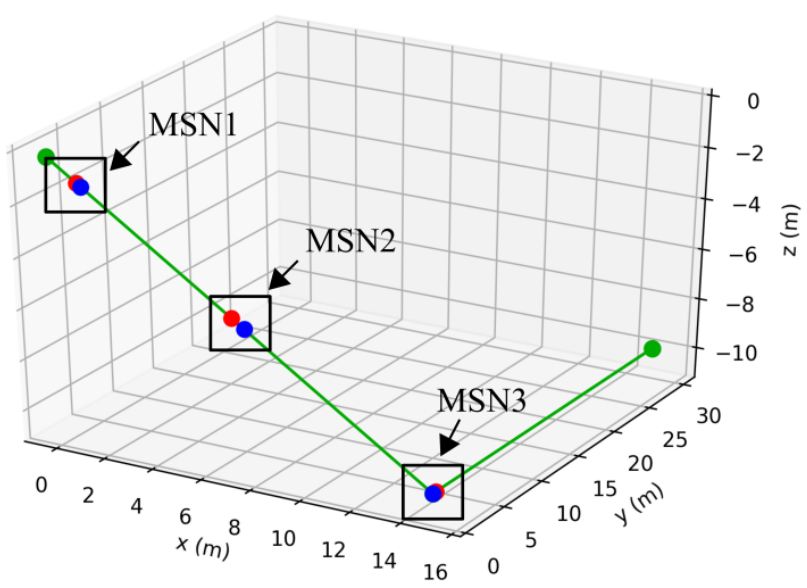

Fig. 8. Comparison of the Convergence Position of each MSN by Evaluation Method. The Red Dots are the Convergence Coordinates of each MSN by the Proposed Method, the Blue Dots are the Convergence Coordinates of each MSN by the Proposed Method (the Remaining Energy is not Evaluated).

\section{DISCUSSION}

The simulations demonstrated that the proposed method could place multiple MSNs in a location where RSSI maintenance and energy consumption were balanced, including an environment with elevation differences. Thus, this disaster 
information-gathering support system can simultaneously achieve high reliability of communication connectivity and long-term operation. The RSSI observation resulted in instantaneous fluctuations due to noise. The MSN moves when the RSSI value at the time is less than the threshold. However, the MSN is at the position where the RSSI can be maintained without movement. As this movement is unnecessary, considering it might be inefficient in terms of energy and movement efficiency. Further, if the MSN is placed by considering the communication quality, noise countermeasures are necessary.

The moving energy consumption of the MSN1 increased because the operation load was concentrated on the MSN1 by the proposed method. The remaining energy of each MSN was balanced as a result. Moreover, since the comparison of the convergence positions of each MSN hardly changed, the reliability of energy consumption experiment was presented. Equation (1) can be used when the network topology is linear. In other words, if the network topology changes during the construction of the WSN, it is necessary to change (1) accordingly. Thus, the method of deploying multiple MSNs can solve two problems in this information-gathering system that maintains communication connectivity and operates for a long time by evaluating communication quality and energy.

\section{CONCLUSION}

This paper proposed a method of operating a robot and multiple MSNs in a disaster area information collection support system while considering communication quality and operating status. While collecting environment information using a robot, the information is represented by a topological map and output as the MSNs' moving route information. In addition, MSN was operated by evaluating RSSI and the remaining energy. The experimental results confirmed that deployment is possible in an environment with a difference in elevation by utilizing movement path information. Therefore, this strategy constructs stable WSNs that can operate for a long time using a rescue robot. The strategy is effective for gathering disaster-area information in actual disaster scenarios. The proposed strategy will be applied for WSN deployment in an actual underground space in the future.

\section{ACKNOWLEDGMENT}

We would like to thank Editage (www.editage.com) for English language editing.

\section{REFERENCES}

[1] H. Kawakata, Y. Kawata, H. Hayashi, T. Tanaka, K. C. Topping, K. Yamori, P. Yoshitomi, and T. Kugai, "Building an integrated database management system of information on disaster hazard, risk, and recovery process," Annuals of Disas. Prev. Res. Inst., Kyoto Univ., No. $47 \mathrm{C}, 2004$.

[2] Y. Kawata, "Disaster mitigation due to next Nankai earthquake tsunamis occurring in around 2035," Proceedings of International Tsunami Symposium 2001, session 1, pp. 315-329, 2001.

[3] Y. Kawata, "The great Hanshin-Awaji earthquake disaster: damage, social response, and recovery," Journal of Natural Disaster Science, vol. 17, no. 2, pp. 1-12, 1995.

[4] M. Erdelj, E. Natalizio, K. R. Chowdhury, and I. F. Akyildiz, "Help from the Sky: Leveraging UAVs for Disaster Management," IEEE Pervasive Computing, vol. 16, no. 1, pp. 24-32, 2017.
[5] W. Shan, J. Feng, J. Chang, F. Yang, and Z. Li, "Collecting earthquake disaster area information using smart phone," 2012 International Conference on System Science and Engineering (ICSSE), Dalian, Liaoning, pp. 310-314, 2012.

[6] T. Gurkan, G. V. Cagri, and G. Kayhan, "An autonomous wireless sensor network deployment system using mobile robots for human existence detection in case of disasters," Ad Hoc Networks, vol. 13, pp. 54-68, 2014.

[7] A. Mangla, A. K. Bindal, and D. Parasad, "Disaster Management in wireless sensor networks: A survey report," International Journal of Computing and Corporate Research, vol. 6, 2016.

[8] K. Sawai, T. Suzuki, H. Kono, Y. Hada, and K. Kawabata, "Development of a SN with impact-resistance capability for gathering disaster area information," 2008 International Symposium on Nonlinear Theory and its Applications (NOLTA2008), pp. 17-20, 2008.

[9] T. Suzuki, R. Sugizaki, K. Kawabata, Y. Hada, and Y. Tobe, "Autonomous deployment and restoration of sensor network using mobile robots," International Journal of Advanced Robotic System, vol. 7, no. 2, pp. 105-114, 2010.

[10] K. Sawai, S. Tanabe, H. Kono, Y. Koike, R. Kunimoto, and T. Suzuki, "Construction strategy of wireless sensor networks with throughput stability by using mobile robot," International Journal of Advanced Computer Science and Applications, The Science and Information organization, vol. 5, no. 2, pp. 14-20, 2014.

[11] M. Rajesh, A. George, and T. S. B. Sudarshan, "Energy efficient deployment of wireless sensor network by multiple mobile robots," 2015 International Conference on Computing and Network Communications (CoCoNet), pp. 72-78, 2015.

[12] Z. Hao, N. Qu, X. Dang, and J. Hou, "RSS-based coverage deployment method under probability model in 3D-WSN," in IEEE Access, vol. 7, pp. 183091-183104, 2019.

[13] T. Saitou, M. Nukada, and Y. Uchimura, "Deployment control of mobile robots for wireless network relay based on received signal strength," 2013 IEEE Workshop on Advanced Robotics and its Social Impacts, pp. 237-242, 2013.

[14] X. Zhong and Y. Zhou, "Maintaining wireless communication coverage among multiple mobile robots using Fuzzy Neural Network," IEEE/ASME International Conference on Mechatronics and Embedded Systems and Applications (MESA), pp. 35-41, 2012.

[15] J.Wang, C. Ju, Y. Gao, A. K. Sangaiah, and G. J. Kim, "A PSO based energy efficient coverage control algorithm for wireless sensor networks," Comput. Mater. Contin, Vol. 56, No. 3, pp. 433-446, 2018.

[16] M. Bakshi, A. Ray, and D. De, "Maximizing lifetime and coverage for minimum energy wireless sensor network using corona based sensor deployment," CSI transactions on ICT, Vol. 5, No. 1, pp. 17-25, 2017.

[17] Y. Li, B. Zhang, and S. Chai, "An energy balanced-virtual force algorithm for Mobile-WSNs," 2015 IEEE International Conference on Mechatronics and Automation (ICMA), pp. 1779-1784, 2015.

[18] S. Halder and A. Ghosal, "Is sensor deployment using Gaussian distribution energy balanced?" 2014 IEEE 11th Consumer Communications and Networking Conference (CCNC), pp. 721-728, 2014.

[19] J. P. Godard, "Urban underground space and benefits of going underground," Proc. World Tunnel Congress 2004 and 30th ITA General Assembly, Singapore, pp. 1-9. May 22-27 , 2004.

[20] H. Cheng, H. Chen, and Y. Liu, "Topological indoor localization and navigation for autonomous mobile robot," IEEE Transactions on Automation Science and Engineering, vol. 12, no. 2, pp. 729-738, 2015.

[21] Y. Kato, K. Kamiyama, and K. Morioka, "Autonomous robot navigation system with learning based on deep Q-network and topological maps," 2017 IEEE/SICE International Symposium on System Integration (SII), pp. 1040-1046, 2017.

[22] E. W. Dijkstra, "A note on two problems in connexion with graphs," Numerische Mathematik, Vol. 1, No. 1, pp. 269-271, 1959.

[23] ns-3 Network Simulator, https://www.nsnam.org/ (Accessed 2020-2-18) 